\title{
Nonuniformly Spaced Linear Antenna Array Design Using Firefly Algorithm
}

\author{
Mohammad Asif Zaman and Md. Abdul Matin \\ Department of Electrical and Electronic Engineering, Bangladesh University of Engineering and Technology, Dhaka 1000, Bangladesh \\ Correspondence should be addressed to Mohammad Asif Zaman, asifzaman13@gmail.com
}

Received 31 October 2011; Revised 23 January 2012; Accepted 31 January 2012

Academic Editor: Ramon Gonzalo

Copyright ( $) 2012$ M. A. Zaman and Md. Abdul Matin. This is an open access article distributed under the Creative Commons Attribution License, which permits unrestricted use, distribution, and reproduction in any medium, provided the original work is properly cited.

\begin{abstract}
A nonuniformly spaced linear antenna array with broadside radiation characteristics is synthesized using firefly algorithm and particle swarm optimization. The objective of the work is to find the optimum spacing between the radiating antenna elements which will create a predefined arbitrary radiation pattern. The excitation amplitudes of all the antenna elements are assumed to be constant. The optimum spacing between the array elements are obtained using firefly algorithm. The minimum allowed distance between the antenna elements is defined in such a way that mutual coupling between the elements can be ignored. Numerical analysis is performed to calculate the far-field radiation characteristics of the array. Two numerical examples are shown to form two different desired predefined radiation patterns. The performance of the firefly algorithm and particle swarm optimization is compared in terms of convergence rate and global best solution achieved. The performances of the optimized nonuniformly spaced arrays are analyzed. Finally, contour plots of the radiated power from the optimized array in the horizontal plane and vertical plane in the far-field region are provided.
\end{abstract}

\section{Introduction}

Multiple antennas can be arranged in space in various geometrical configurations to form an antenna array with highly directive radiation pattern $[1,2]$. The radiation characteristics of the antenna array depend on some input parameters. These parameters are the relative magnitude and phase of the excitation current of each radiating element, radiation characteristics of each radiating element, the geometrical configuration of the array, and the separation distance between the array elements [2]. An antenna array can be designed to produce almost any arbitrary prescribed pattern by controlling these parameters. For this reason, antenna arrays find application in RADAR and wireless communication systems $[3,4]$.

Most antenna arrays are designed to produce a directive beam at a particular direction and while keeping the sidelobe level (SLL) small to avoid interference with other radiating sources. In most cases, this is achieved by controlling the magnitude and phase of the excitation amplitudes $[5,6]$. In most cases, a relatively simple geometry is considered where the distance between two consecutive radiating elements is constant. However, exact control of phase and magnitude of excitation current of array elements requires complex and expensive electronic circuitry [4]. In case of phased arrays, where the direction of the main beam needs to be controlled electronically in real time [7], uses of such electronic circuits are unavoidable. However, in many applications, a dynamic control of array radiation pattern is not required. It is highly desirable in such cases to design an antenna array that does not require complex circuitry to control the phase and magnitude of excitation currents. Desired radiation characteristics can be achieved by proper placement of each individual radiating element in space while keeping the excitation current constant for all elements. Such arrays are known as nonuniformly spaced antenna arrays.

In linear antenna arrays, the antenna elements are placed along a straight line. Linear array of identical radiating 
elements are one of the common type of antenna arrays [4, $6]$. Design method of linear uniformly spaced antenna arrays are widely covered in literature [5-8]. Most of these methods employ a heuristic optimization algorithm to find the phase and/or magnitude of the excitation currents of the array elements while keeping the separation between the array elements uniform. Particle Swarm Optimization (PSO) $[5,7]$, Genetic Algorithm (GA) [6] and Artificial Bee Colony (ABC) algorithm [8] have been successfully used to design such arrays. Although, design methods of Nonuniformly Spaced Linear Antenna arrays (NUSLA) exist, they have not received equal attention in the literature.

One of the first major articles describing nonuniformly spaced antenna arrays was published in 1961 [9]. The design method employed perturbation methods and concentrated on sidelobe reduction. An iterative method for sidelobe reduction was developed by Hodjat and Hovanessian [10]. Recently, Fourier transform and window techniques have been applied for designing NUSLA [11]. These methods are suitable for synthesizing radiation pattern with low SLL, but lacks flexibility to synthesize arbitrary radiation pattern.

Optimization-algorithm-based design methods of NUSLA allowed engineers to easily synthesize arbitrary radiation patters. The applications of DE and GA to synthesize NUSLA for low SLL have been reported in the literature $[12,13]$. PSO has been successfully used to design NUSLA with low SLL and nulls in arbitrary positions [14]. Ant Colony Optimization (ACO) algorithm has also been used to synthesize NUSLA with arbitrary radiation pattern [15].

Oraizi and Fallahpour have presented an impressive study of NUSLA in their paper [16]. They used modified GA-based analysis to optimize the position of the array elements. However, the work does not mention the number of iterations required by GA to converge or any other indication convergence time. GA usually is not fast converging for antenna array problems and it is often outperformed by PSO and $\mathrm{ABC}[7,8]$. In this paper, we used a newly developed optimization algorithm called Firefly Algorithm (FA) to synthesize NUSLA and compare the performance of the algorithm with existing methods.

FA is a heuristic numeric optimization algorithm inspired by the behavior of fireflies $[17,18]$. FA has been successfully used in many applications. Recently, FA has been used by Basu and Mahanti for designing linear antenna arrays [19]. The work concentrated on designing equally spaced linear array with variable excitation current. No work has yet been published on the application of FA for NUSLA design.

In this paper, FA is used to find the optimum spacing between the array elements to produce a desired broadside radiation pattern. The design examples with numerical results are provided. In the first example, a NUSLA is synthesized with minimum possible SLL. In the second example, a NUSLA is synthesized with low SLL and nulls in arbitrary direction. The paper is organized as follows: array geometry and mathematical formulation of the radiated electric field radiated by the array is presented in Section 2. A brief description of FA is given in Section 3. Numerical results are provided in Section 4 and concluding remarks are given in Section 5.

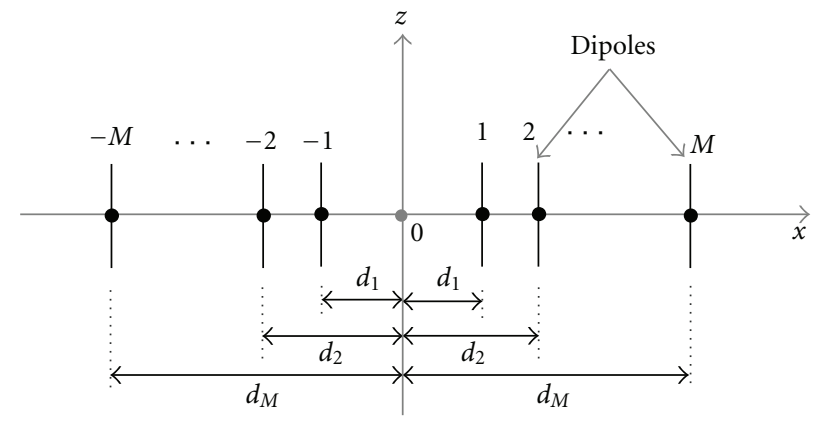

FIGURE 1: Two-dimensional schematic diagram of the array geometry.

\section{Array Geometry and Mathematical Formulation}

The linear antenna array is assumed to be composed of $N$ identical radiating elements. The radiating elements are taken to be dipoles antenna. The dipoles are positioned symmetrically around the origin on the $x$-axis. The arms of the dipoles are parallel to the $z$-axis. A two-dimensional schematic diagram of the array geometry is shown in Figure 1.

The total number of elements, $N$ is assumed to be even. The elements are divided into two groups of $M$ elements where $N=2 M$. The elements are numbered as $-M,-M+$ $1 \cdots-1,1,2, \ldots M-1, M$. The distances of elements $1,2, \ldots M$ from origin is denoted by $d_{1}, d_{2}, \ldots d_{M}$. Due to symmetry, $-1,-2, \ldots-M$ elements have the same distance values. The symmetry around the origin creates symmetrical radiation pattern, which is often desirable. It is possible to create symmetrical radiation pattern with unsymmetrical positioning of the array elements. However, the symmetry condition reduces computational complexities, as now only position of $M$ elements must be optimized instead of position of $N$ elements. To avoid complexities, symmetrical distribution of the elements around the origin is assumed in this paper.

The radiated field of the array depends on the radiation pattern of each array element and relative spacing of the array elements. The far-field radiation pattern of the array is given by $[1,3]$

$$
\operatorname{FF}(\theta, \phi)=\operatorname{EP}(\theta, \phi) \times \operatorname{AF}(\theta, \phi) .
$$

Here, $\operatorname{EP}(\cdot)$ is the radiation pattern of individual array elements, $\operatorname{AF}(\cdot)$ is the array factor, and $(\theta, \phi)$ are the zenith and azimuth angle of the spherical coordinate system. The coordinate system and the three dimensional geometry are shown in Figure 2.

The radiation pattern of each array element is assumed to be

$$
\mathrm{EP}(\theta, \phi)=\sin \theta
$$

The array factor is given by [16]

$$
\operatorname{AF}(\theta, \phi)=\frac{1}{N} \sum_{n=1}^{M} \cos \left(k d_{n} \cos \phi\right) .
$$




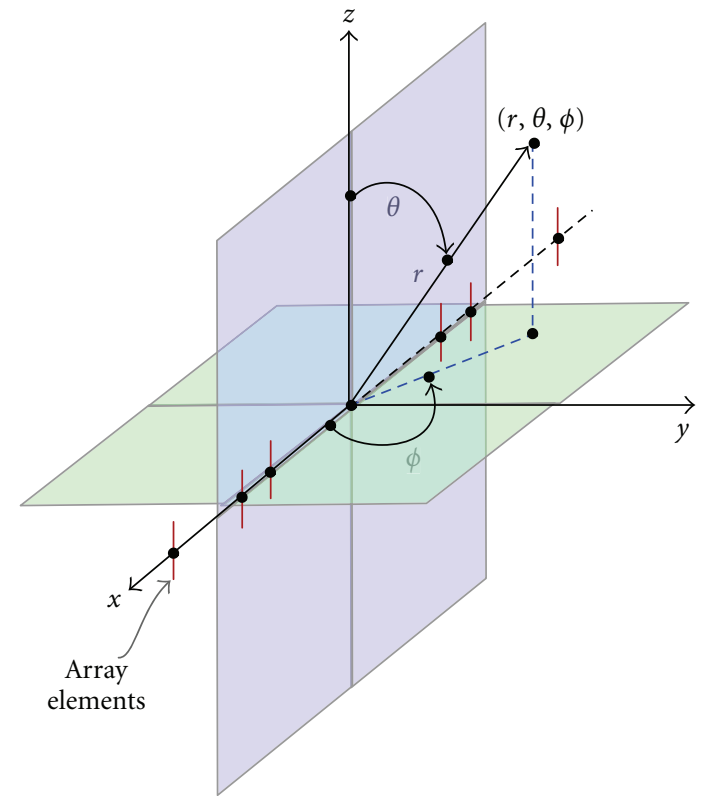

Figure 2: Coordinate system and three dimensional geometry of the array.

Here, $k$ is wave number $=2 \pi / \lambda$ and $\lambda$ is wavelength of radiation.

Using (1), (2), and (3), the overall radiation pattern is given by

$$
\mathrm{FF}(\theta, \phi)=\sin \theta\left[\frac{1}{N} \sum_{n=1}^{M} \cos \left(k d_{n} \cos \phi\right)\right] .
$$

In this paper, the objective is to find optimum values of $d_{n}$ to produce a desired far-field radiation pattern. It is noted that for the given geometry and type of radiating elements chosen, $d_{n}$ does not affect the field variation with $\theta$. So, for optimization process is carried out at $\theta=90^{\circ}$ plane only.

Desired radiation pattern must be defined to measure the performance of the array. Since two different designs are to be implemented using the proposed method, two different desired patterns are defined as follows:

$$
\begin{gathered}
\mathrm{FF}_{\mathrm{des}, 1}\left(90^{\circ}, \phi\right)= \begin{cases}0 \mathrm{~dB}, & -\frac{\mathrm{BW}}{2}<\phi<\frac{\mathrm{BW}}{2} \\
\mathrm{SLL}_{\mathrm{des}}, & \text { otherwise. }\end{cases} \\
\mathrm{FF}_{\mathrm{des}, 2}\left(90^{\circ}, \phi\right)= \begin{cases}0 \mathrm{~dB}, & -\frac{\mathrm{BW}}{2}<\phi<\frac{\mathrm{BW}}{2}, \\
\mathrm{NLL}_{\text {des }}, & \frac{\phi_{\mathrm{NL}}}{2}<\phi<\frac{\phi_{\mathrm{NH}}}{2}, \\
\mathrm{SLL}_{\text {des }}, & \text { otherwise. }\end{cases}
\end{gathered}
$$

Here, BW is desired main beamwidth, $\mathrm{SLL}_{\mathrm{des}}$ is desired sidelobe level, $\mathrm{NLL}_{\mathrm{des}}$ is desired null level, $\phi_{\mathrm{NS}}$ is desired starting angular position of the null, and $\phi_{\mathrm{NE}}$ is desired ending angular position of the null.

Equation (5) represents a desired radiation pattern with a prescribed SLL and beamwidth. Equation (6) represents a desired radiation pattern with a specific SLL and beamwidth along with a predefined null level at an arbitrary direction. The angular extent of the null region is defined by the angles $\phi_{\mathrm{NS}}$ and $\phi_{\mathrm{NE}}$.

The deviation of the obtained far-field pattern from the desired pattern is evaluated using the cost function. The cost function is defined as

$$
f_{\cos t}=\left\{\begin{array}{l}
\sum_{\phi=0^{\circ}}^{180^{\circ}}\left\{\mathrm{FF}\left(90^{\circ}, \phi\right)-\mathrm{FF}_{\mathrm{des}}\left(90^{\circ}, \phi\right)\right\}^{2}, \\
\mathrm{FF}\left(90^{\circ}, \phi\right)>\mathrm{FF}_{\mathrm{des}}\left(90^{\circ}, \phi\right), \\
0, \quad \text { otherwise. }
\end{array}\right.
$$

Here, the summation is performed over the discrete field points that are numerically calculated. For the two different desired patterns defined in (5) and (6), two different cost functions can be defined. For each case, the fitness function is defined as

$$
f_{\text {fitness }}=-f_{\cos t} \text {. }
$$

It can be noted that, when the obtained radiation pattern matches with the desired pattern, the cost function and the fitness function have zero values. When the patterns do not match, the cost function has positive values and the fitness function has negative values. Large deviations result is high positive cost values and high negative fitness values. So, the goal of the optimization process is to minimize the cost function or maximize the fitness function by finding appropriate values of $d_{n}$.

\section{Overview of Firefly Algorithm}

FA is an optimization algorithm inspired by behavior and motion of fireflies. It is a population-based optimization algorithm which uses swarm intelligence to converge $[17,18]$. It is similar to other optimization algorithms employing swarm intelligence such as PSO and ABC. But FA is found to have superior performance in many cases [19].

FA initially produces a swarm of fireflies located randomly in the search space. The initial distribution is usually produced from a uniform random distribution. The position of each firefly in the search space represents a potential solution of the optimization problem. The dimension of the search space is equal to the number of optimizing parameters in the given problem. The fitness function takes the position of a firefly as input and produces a single numerical output value denoting how good the potential solution is. A fitness value is assigned to each firefly. The FA uses a phenomenon known is bioluminescent communication to model the movement of the fireflies through the search space. The brightness of each firefly depends on the fitness value of that firefly. Each firefly is attracted by the brightness of other fireflies and tries to move towards them. The velocity or the pull a firefly towards another firefly depends on the attractiveness. The attractiveness depends on the relative distance between the fireflies. It can be a function of the brightness of the fireflies as well. A brighter firefly far away may not be as attractive as a less bright firefly that is closer. In each iterative 
step, FA computes the brightness and the relative attractiveness of each firefly. Depending on these values, the positions of the fireflies are updated. After a sufficient amount of iterations, all fireflies converge to the best possible position on the search space.

The number of fireflies in the swarm is known as the population size, $P$. The selection of population size depends on the specific optimization problem. However, typically a population size of 20 to 40 is used for PSO and FA for most applications $[14,17]$. For the current problem, the solution space is $M$ dimensional, where each dimension represents the position of an array element. The position of the $n$th firefly is denoted by a vector $\mathbf{x}_{\mathbf{n}}$ where,

$$
\mathbf{x}_{\mathbf{n}}=\left(x_{n}^{1}, x_{n}^{2}, x_{n}^{3}, \ldots, x_{n}^{m}, \ldots, x_{n}^{M}\right) .
$$

Here, $n=1,2,3 \cdots P$ and $m=1,2,3, \ldots, M$.

The search space is limited in mth dimension by the following inequality:

$$
x_{\text {Low }}^{m}<x_{n}^{m}<x_{\text {High }}^{m} .
$$

The value of the variables $x_{\text {Low }}^{m}$ and $x_{\text {High }}^{m}$ depend on the optimization problem. For the current NUSLA synthesis problem, these variables represent minimum allowed and maximum allowed separation distance of consecutive array elements. Initially, the positions of the fireflies are generated from a uniform distribution using the following equation:

$$
x_{n}^{m}=x_{\text {Low }}^{m}+\left(x_{\text {High }}^{m}-x_{\text {Low }}^{m}\right) \times \text { rand } .
$$

Here, rand is a uniform random variable with values ranging from 0 to 1 . The value of rand is different for each value of $m$ and $n$. Equation (11) generates random values from a uniform distribution within the prescribed range defined by (10). The initial distribution does not significantly affect the performance of the algorithm. Each time the algorithm is executed, the optimization process starts with a different set of initial points. However, in each case, the algorithm finds the optimum solution. In case of multiple possible sets of solutions, the algorithm may converge on different solutions each time. But each of those solutions will be valid as they all will satisfy the design requirements.

The brightness of the $n$th firefly, $I_{n}$ is given by

$$
I_{n}=f_{\text {fitness }}\left(\mathbf{x}_{\mathbf{n}}\right)
$$

The attractiveness between the $n$th and pth firefly, $\beta_{n p}$ given by [17]

$$
\beta_{n p}=\beta_{o} \exp \left(-\gamma r_{n p}^{2}\right)
$$
by

Here, $r_{n p}$ is Cartesian distance between $\mathbf{x}_{\mathbf{n}}$ and $\mathbf{x}_{\mathbf{p}}$ given

$$
r_{n p}=\left\|\mathbf{x}_{\mathbf{n}}-\mathbf{x}_{\mathbf{p}}\right\|=\sqrt{\sum_{m=1}^{M}\left(x_{n}^{m}-x_{p}^{m}\right)^{2}} .
$$

$\beta_{o}$ is a constant taken to be $1 . \gamma$ is another constant whose value is related to the dynamic range of the search space.

The position of firefly is updated in each iterative step. If the brightness of pth firefly is larger than the brightness of the $n$th firefly, then the $n$th firefly moves towards the pth firefly. The motion is denoted by the following equation:

$$
\mathbf{x}_{\mathbf{n}, \text { new }}=\mathbf{x}_{\mathbf{n}, \text { old }}+\beta_{m n}\left(\mathbf{x}_{\mathbf{p}, \text { old }}-\mathbf{x}_{\mathbf{n}, \text { old }}\right)+\alpha(\text { rand }-0.5) .
$$

Here, rand is a random number between 0 and 1 , taken from a uniform distribution. $\alpha$ is a constant whose value depends on the dynamic range of the solution space.

At each iterative step, the brightness and the attractiveness of each firefly is calculated. The brightness of each firefly is compared with all other fireflies and the positions of the fireflies are updated using (15). After a sufficient number of iterations, all the fireflies converge to the same position in the search space and the global optimum is achieved.

\section{Numerical Simulation and Results}

For numerical simulations, a $N=20$ element NUSLA is considered. As symmetry about the origin is assumed, only the position of $M=10$ elements located on the positive $x$ axis needs to be optimized. The population size is taken to be 40 and maximum number of iterations is limited to 65 . The search space is bounded by defining the minimum and maximum separation between two consecutive elements to be $0.35 \lambda$ and $0.9 \lambda$, respectively, implying the solution space limiting variables defined in (10) have the following values:

$$
\begin{aligned}
& x_{\mathrm{Low}}^{m}=0.35 \lambda, \\
& x_{\mathrm{High}}^{m}=0.9 \lambda .
\end{aligned}
$$

For $m=1,2, \ldots, M$. The limiting values in all dimensions of the solution space are assumed to be the same. These values are selected so that mutual coupling between the elements remains negligible [16]. The dynamic range of the search space is $0.9 \lambda-0.35 \lambda=0.55 \lambda$. $\alpha$ is taken to be $80 \%$ of the dynamic range initially and the value is linearly decreased to zero at maximum iteration number. The parameter $\gamma$ is taken to be equal to the dynamic range. So,

$$
\begin{gathered}
\alpha=0.8 \times\left(x_{\mathrm{High}}^{m}-x_{\mathrm{Low}}^{m}\right), \\
\gamma=x_{\mathrm{High}}^{m}-x_{\mathrm{Low}}^{m} .
\end{gathered}
$$

The parameters of the first desired radiation pattern defined by (5) are selected as

$$
\begin{gathered}
\mathrm{BW}<13.4^{\circ} \\
\mathrm{SLL}_{\mathrm{des}}=-23.5 \mathrm{~dB} .
\end{gathered}
$$

These values are selected based on typical beamwidth and SLL values of 20 element linear antenna array [16]. From multiple trial runs of the optimization algorithm, it was verified that these were the lowest possible values of beamwidth and SLL. 
TABLE 1: Optimized position of the array elements for the first design example.

\begin{tabular}{llllllllllll}
\hline \multicolumn{2}{r}{ Element number, $n$} & 1 & 2 & 3 & 4 & 5 & 6 & 7 & 8 & 9 & 10 \\
\hline \multirow{2}{*}{ Position, $d_{n} / \lambda$} & FA & 0.189 & 0.540 & 0.934 & 1.292 & 1.726 & 2.141 & 2.639 & 3.173 & 3.899 & 4.634 \\
& PSO & 0.239 & 0.857 & 1.362 & 1.929 & 2.576 & 3.193 & 3.881 & 4.762 & 5.662 & 6.397 \\
\hline
\end{tabular}

The parameters of the second desired radiation pattern defined by (6) are selected as

$$
\begin{gathered}
\mathrm{BW}<12^{\circ} \\
\mathrm{SLL}_{\mathrm{des}}=-20 \mathrm{~dB} \\
\mathrm{NLL}_{\mathrm{des}}=-40 \mathrm{~dB} \\
\phi_{\mathrm{NS}}=46^{\circ}, 126^{\circ} \\
\phi_{\mathrm{NE}}=54^{\circ}, 134^{\circ} .
\end{gathered}
$$

Two nulls symmetrical spaced around the main beam (located at $90^{\circ}$ ) extending from $46^{\circ}$ to $54^{\circ}$ and $126^{\circ}$ to $134^{\circ}$ with null level of $-40 \mathrm{~dB}$ are desired. To achieve this, the requirements on desired SLL must be relaxed compared to the previous case. For this reason, desired SLL in this case is set to $-20 \mathrm{~dB}$ compared to previous value of $-23 \mathrm{~dB}$. This relaxation in SLL constraint allows the possibility of reduction of main beamwidth. For this reason, the maximum main beamwidth in this case is selected as $12^{\circ}$ compared to $13.4^{\circ}$ of the previous case.

The NUSLA for the two desired radiation patterns is designed using FA algorithm. For comparison, the same designs are performed using PSO algorithm also. The value of the parameters of the PSO algorithm is selected the same as the ones used in [14]. However, the population size of 20 was used in [14]. For proper comparison, the population size for the PSO algorithm is taken to be 40, which is identical to the population size used in the FA algorithm.

FORTRAN computer coding (with G95 compiler) is used to implement the FA and PSO algorithm and formulating the far-field pattern. The optimum position of the array elements obtained from FA and PSO for the first desired radiation pattern is shown in Table 1.

It can be observed from Table 1 that the two algorithms produce different sets of solution values. Using these values, the far-field radiation pattern at $\theta=90^{\circ}$ plane ( $x y$ plane) is calculated. The radiation pattern obtained from the FA is shown in Figure 3 and the radiation pattern obtained from the PSO algorithm is shown in Figure 4. The desired pattern is also highlighted in red in the same figures.

It can be seen that both radiation patterns satisfy most of the design criterion. In [16], the maximum SLL of $-22.6 \mathrm{~dB}$ was achieved with a 20 element NUSLA of similar geometry. In this paper, using FA algorithm, the SLL value is limited to $-23.5 \mathrm{~dB}$ using the same number of array elements. So the proposed FA-based outperforms the GA-based method described in [16]. For the PSO algorithm, SLL constraint has not been met by the design obtained from PSO in only a small angular region. So, FA outperforms PSO in terms of output as well. However, the performance of the algorithms cannot be compared form the final output only. The convergence speed of the algorithms can show the difference in performance: the convergence rate observed from the fitness

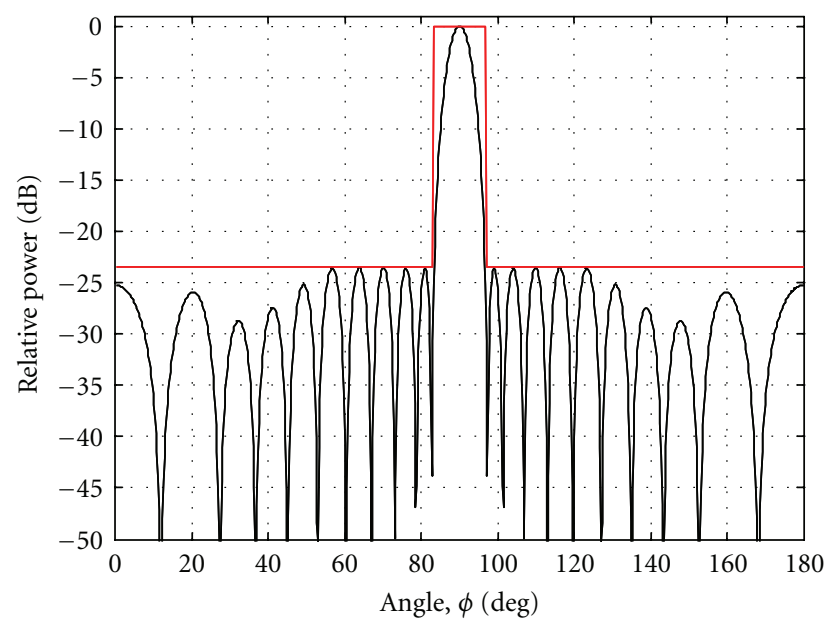

FIgure 3: Optimized radiation pattern of the NUSLA at $\theta=90^{\circ}$ plane obtained from FA algorithm for the first design example.

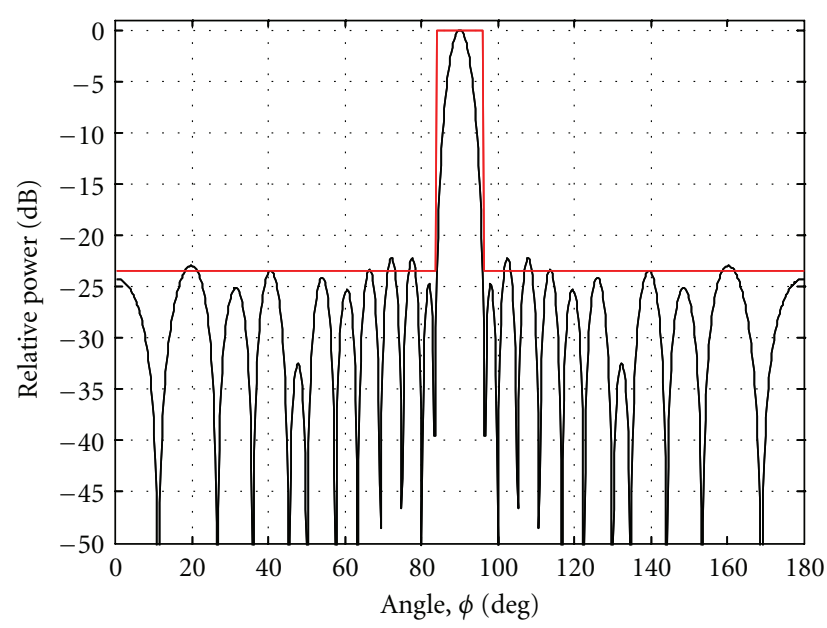

FIgURE 4: Optimized radiation pattern of the NUSLA at $\theta=90^{\circ}$ plane obtained from PSO algorithm for the first design example.

versus iteration plot for the algorithms. This is shown in Figure 5.

It can be seen that for FA, the fitness function value reaches its maximum possible value zero within 20 iterations. The saturation of fitness value implies convergence. However, for PSO, maximum value of -14.317 is achieved. It took PSO around 50 iterations to reach this value. The fact that zero fitness value was not achieved implies that the obtained radiation pattern does not perfectly match with the desired radiation pattern, which is apparent from Figure 4. The most noticeable fact observed from Figure 5 is the fast converging characteristics of FA compared to PSO indicated 
TABLE 2: Optimized position of the array elements for the second design example.

\begin{tabular}{llllllllllll}
\hline \multicolumn{2}{r}{ Element number, $n$} & 1 & 2 & 3 & 4 & 5 & 6 & 7 & 8 & 9 & 10 \\
\hline \multirow{2}{*}{ Position, $d_{n} / \lambda$} & FA & 0.214 & 0.689 & 1.087 & 1.504 & 2.018 & 2.578 & 3.045 & 3.805 & 4.449 & 5.106 \\
& PSO & 0.219 & 0.654 & 1.125 & 1.539 & 2.176 & 2.666 & 3.236 & 3.950 & 4.478 & 5.083 \\
\hline
\end{tabular}

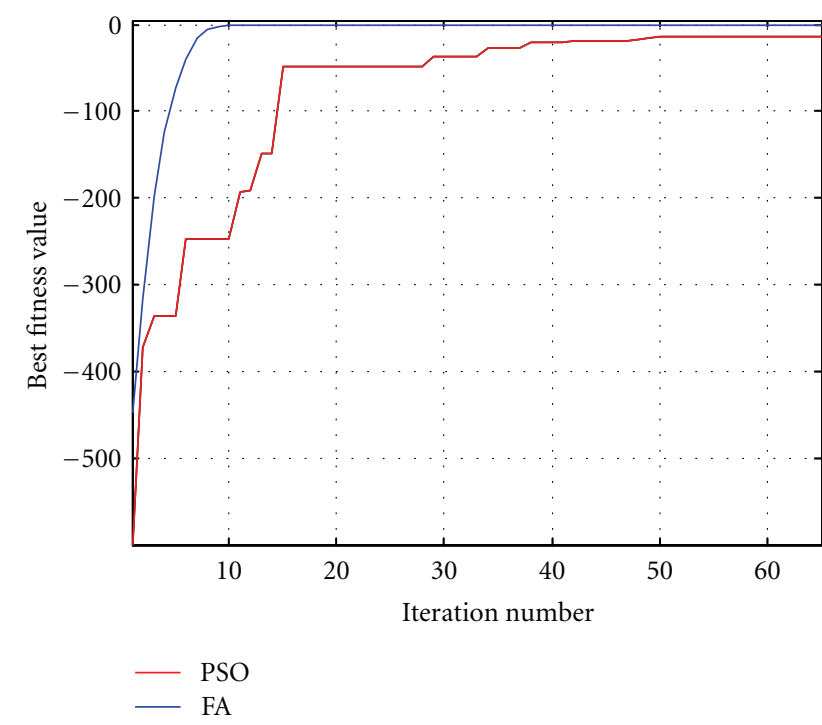

FIGURe 5: Fitness value versus iteration number for FA and PSO algorithm for the first design problem.

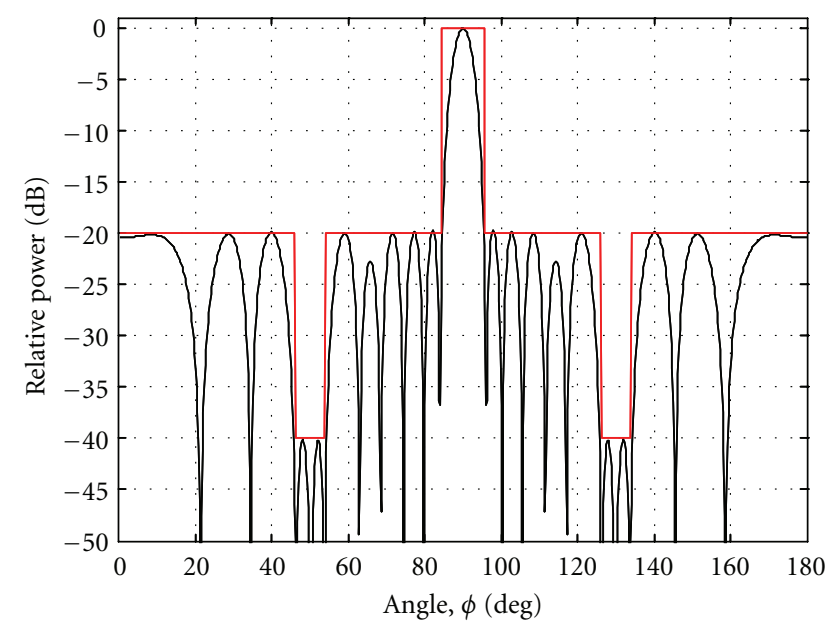

FIgURE 6: Optimized radiation pattern of the NUSLA at $\theta=90^{\circ}$ plane obtained from FA algorithm for the second design example.

by the sharper slope of the blue curve compared to the red one.

Similar analysis was performed to synthesize NUSLA for the second desired radiation pattern. The second desired pattern is characterized by two nulls located symmetrically around the main-lobe region. The optimum position of the array elements obtained from FA and PSO for the first desired radiation pattern is shown in Table 2.

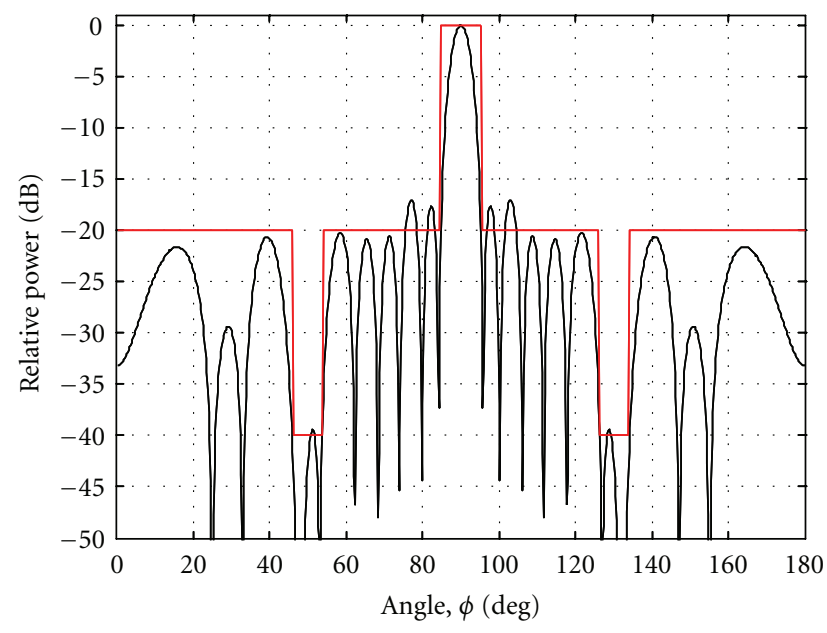

FIGURE 7: Optimized radiation pattern of the NUSLA at $\theta=90^{\circ}$ plane obtained from PSO algorithm for the second design example.

It can be observed from Table 2 that the two algorithms produce different sets of solution values. The radiation pattern obtained from the FA and PSO algorithm are shown in Figures 6 and 7, respectively. The desired pattern is also highlighted in red in the same figures.

It can be observed that the results obtained from FA satisfy the design criterion very well. The results from PSO maintain the desired pattern for the most part, with only a few sidelobes exceeding the limit. This implies that PSO has not converged perfectly. This fact can be further illustrated by observing the fitness function values. This is shown in Figure 8. Again, the superior performance of FA compared to PSO can be observed by the sharper slope of the blue curve compared to the red curve. The maximum fitness value achieved for FA is -0.1456 , whereas this value is -82.223 . FA clearly outperforms PSO in terms of convergence rate and maximum fitness value reached within a limited number of iterations.

Due to the presence of random number parameters in FA and PSO, the results vary on each time the program is executed. However, in all cases, the FA converges faster than PSO. Since, PSO usually outperforms GA for antenna array problems, it is expected that FA will outperform GA as well. ABC usually requires over 100 iterations (with a population size of 30 to 40 ) to reach convergence in antenna array problems $[7,8]$. So, it can be concluded that FA is a very fast converging algorithm.

Using the optimized position of the array elements, the normalized radiated power in the far-field zone of the antenna array is calculated for the first design problem. Equation (4) along with the fact that power varies at $1 / r^{2}$ is 


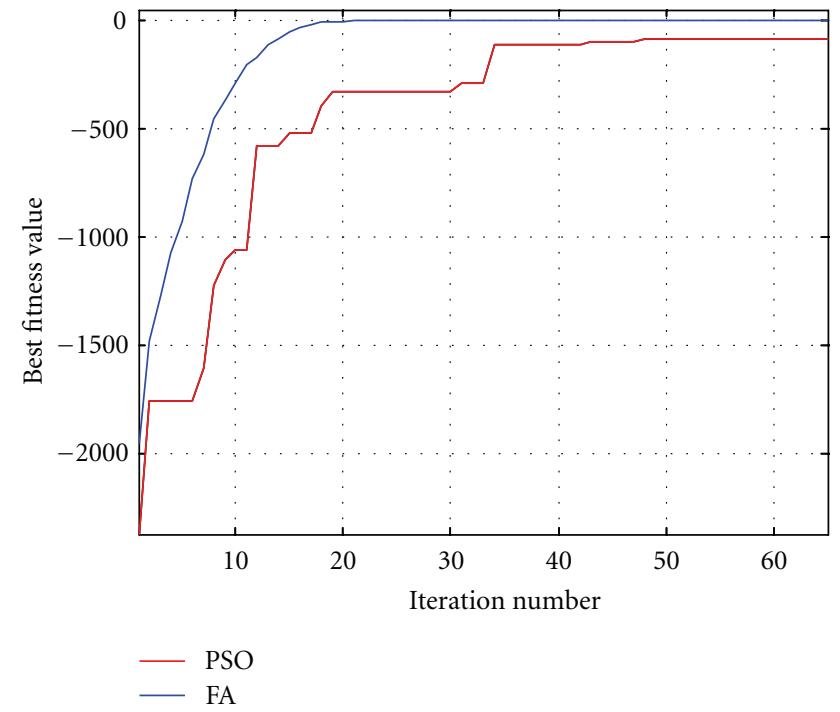

Figure 8: Fitness value versus iteration number for FA and PSO algorithm for the second design problem.

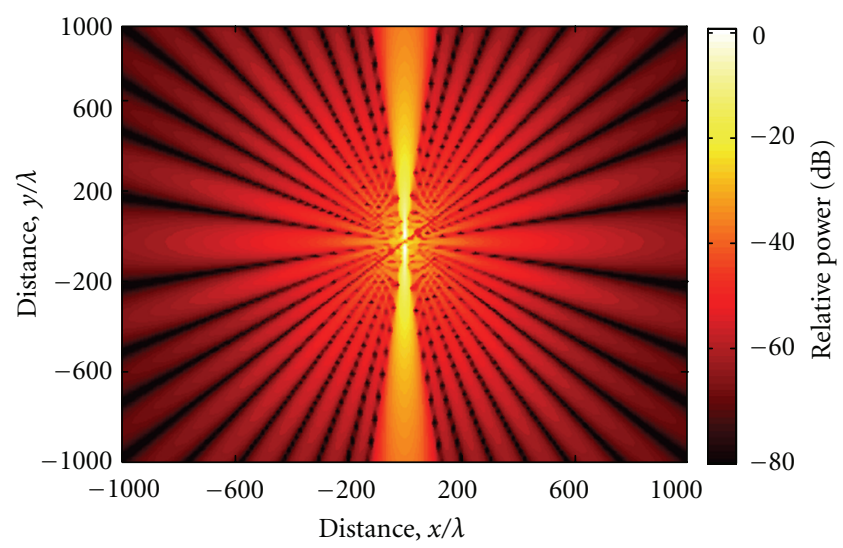

FIgURE 9: Relative power at far-field region in the $x y$ plane.

used to calculate contour plots of normalized radiated power at $x y$ plane and $x z$ plane. The plots are shown in Figures 9 and 10 .

As the array elements are located along the $x$ axis, Figure 9 shows the broadside radiation characteristics of the array. The same results are also seen from Figure 3. The power distribution in the $x z$ plane resembles the radiation pattern of a dipole antenna. This is expected, as the array elements are dipoles and the array factor does not contribute to the field distribution at $x z$ plane $\left(\phi=0^{\circ}\right.$ plane $)$.

\section{Conclusion}

Two nonuniformly spaced linear antenna arrays are designed for two distinct design requirements. The radiation characteristics of the designed arrays are numerically evaluated. The separations between the array elements are determined using firefly algorithm and PSO algorithm. It has been found that the design obtained from FA satisfies the predefined SLL,

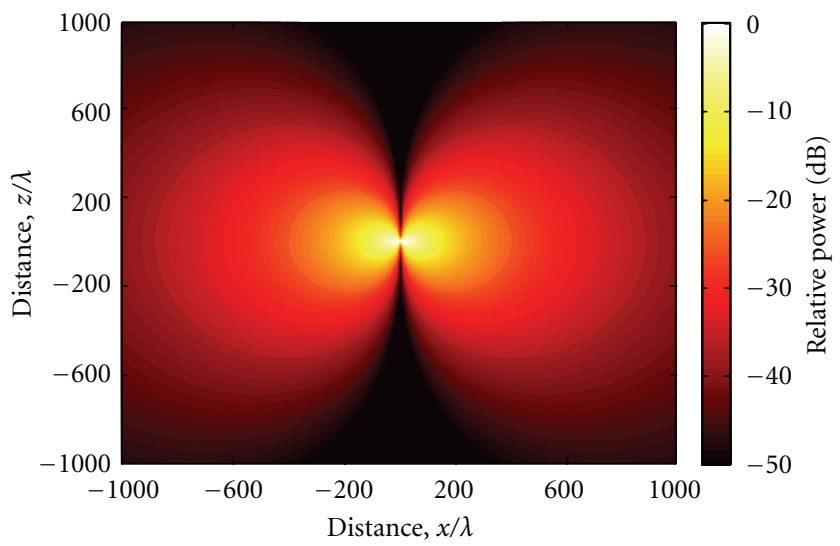

FIGURE 10: Relative power at far-field region in the $x z$ plane.

beamwidth, and null requirements very well. The design obtained from PSO satisfies most of the design requirements but it is outperformed by FA in terms of convergence rate and obtained global best results. Also, a lower value of SLL is achieved using FA-based design method compared to other similar arrays designed by GA. Overall, the FA is found to be very suitable for NUSLA design compared to other existing optimization-algorithm-based methods.

\section{References}

[1] C. A. Balanis, Antenna Theory Analysis and Design, John Wiley \& Sons, 3rd edition, 2005.

[2] C. A. Balanis, Modern Antenna Handbook, John Wiley \& Sons, 2008.

[3] J. D. Kraus, R. J. Marhefka, and A. S. Khan, Antennas for all Applications, Tata McGraw-Hill, 3rd edition, 2007.

[4] R. C. Hansen, "Chapter 20: phased arrays," in Antenna Engineering Handbook, J. L. Volakis, Ed., McGraw-Hill, 4th edition, 2007.

[5] T. B. Chen, Y. B. Chen, Y. C. Jiao, and F. S. Zhang, "Synthesis of antenna array using particle swarm optimization," in Proceedings of the Asia-Pacific Microwave Conference (APMC '05), vol. 3, p. 4, December 2005.

[6] A. Recioui, A. Azrar, H. Bentarzi, M. Dehmas, and M. Chalal, "Synthesis of linear arrays with sidelobe reduction constraint using genetic algorithm," International Journal of Microwave and Optical Technology, vol. 3, no. 5, pp. 524-530, 2008.

[7] M. A. Zaman, S. A. Mamun, M. Gaffar, S. M. Choudhury, and M. A. Matin, "Phased array synthesis using modified particle swarm optimization," Journal of Engineering Science and Technology Review, vol. 4, no. 1, pp. 68-73, 2011.

[8] M. A. Zaman, M. Gaffar, M. M. Alam, S. A. Mamun, and M. A. Matin, "Syntehsis of antenna arrays using artificial bee colony optimization algorithm," International Journal of Microwave and Optical Technology, vol. 6, no. 4, pp. 234-241, 2011.

[9] R. Harrington, "Sidelobe reduction by nonuniform element spacing," IRE Transactions on Antennas and Propagation, vol. 9, no. 2, pp. 187-192, 1961.

[10] F. Hodjat and S. A. Hovanessian, "Nonuniformly spaced linear and planar array antennas for sidelobe reduction," IEEE Transactions on Antennas and Propagation, vol. 26, no. 2, pp. 198204, 1978 . 
[11] X. F. Ren, J. A. Azevedo, and A. M. Casimiro, "Synthesis of non-uniformly spaced arrays using the Fourier transform and window techniques," IET Microwaves, Antennas and Propagation, vol. 3, no. 8, pp. 1245-1253, 2009.

[12] D. G. Kurup, M. Himdi, and A. Rydberg, "Synthesis of uniform amplitude unequally spaced antenna arrays using the differential evolution algorithm," IEEE Transactions on Antennas and Propagation, vol. 51, no. 9, pp. 2210-2217, 2003.

[13] K. Chen, Z. He, and C. Han, "A modified real GA for the sparse linear array synthesis with multiple constraints," IEEE Transactions on Antennas and Propagation, vol. 54, no. 7, pp. 2169 2173, 2006.

[14] M. M. Khodier and C. G. Christodoulou, "Linear array geometry synthesis with minimum sidelobe level and null control using particle swarm optimization," IEEE Transactions on Antennas and Propagation, vol. 53, no. 8, pp. 2674-2679, 2005.

[15] E. Rajo-lglesias and Q. Quevedo-Teruel, "Linear array synthesis using an ant-colony-optimization-based algorithm," IEEE Antennas and Propagation Magazine, vol. 49, no. 2, pp. 70-79, 2007.

[16] H. Oraizi and M. Fallahpour, "Nonuniformly spaced linear array design for the specified beamwidth/sidelobe level or specified directivity/sidelobe level with coupling considerations," Progress in Electromagnetic Research M, vol. 4, pp. 185-209, 2008.

[17] X. S. Yang, "Firefly algorithms for multimodal optimization," in Stochastic Algorithms: Foundations and Appplications, O. Watanabe and T. Zeugmann, Eds., vol. 5792 of SAGA 2009, Lecture Notes in Computer Science, pp. 169-178, Springer, Berlin, Germany, 2009.

[18] X. S. Yang, "Firefly algorithm, stochastic test functions and design optimisation," International Journal of Bio-Inspired Computing, vol. 2, no. 2, pp. 78-84, 2010.

[19] B. Basu and G. K. Mahanti, "Firefly and artificial bees colony algorithm for synthesis of scanned and broadside linear array antenna," Progress in Electromagnetic Research B, vol. 32, pp. 169-190, 2011. 

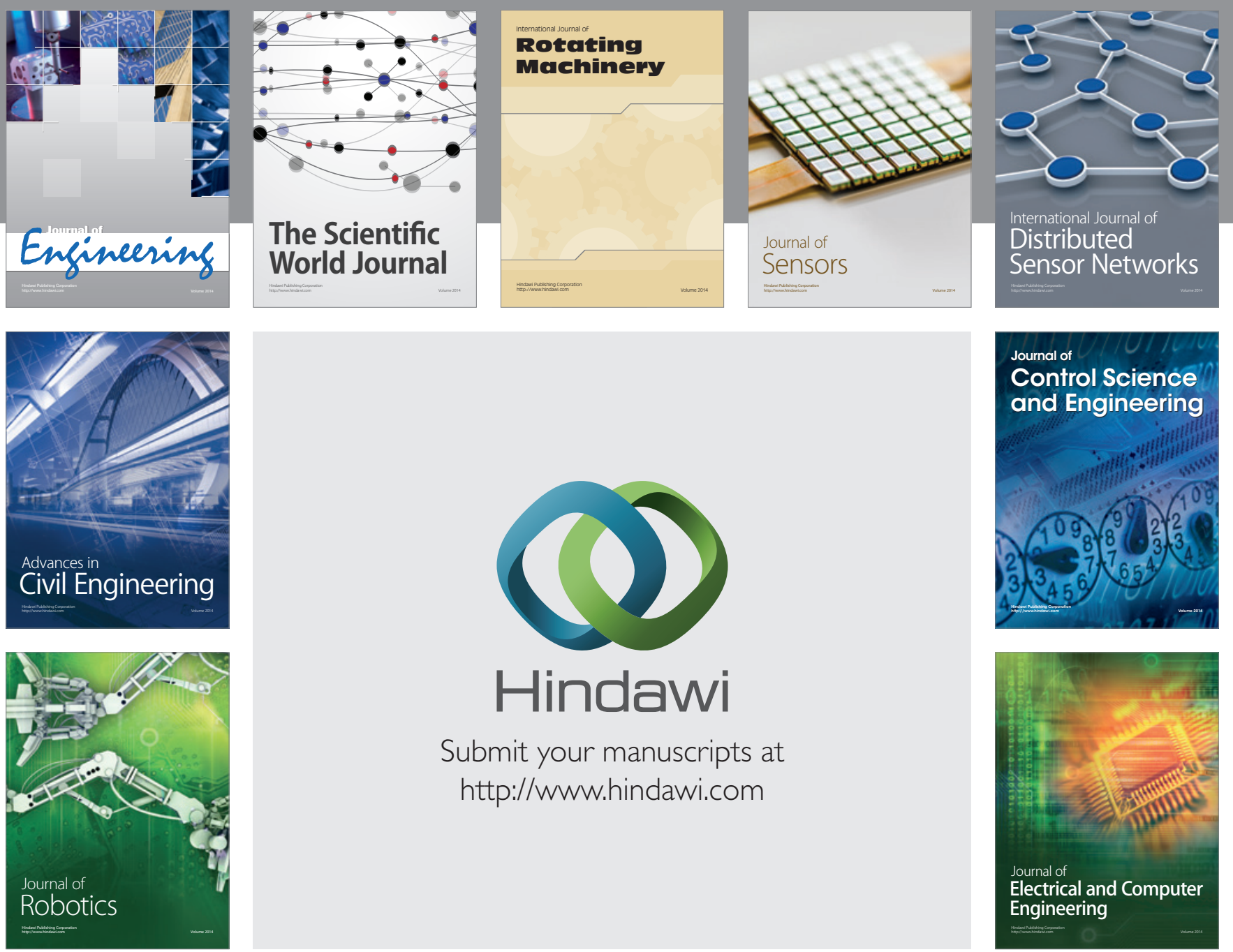

Submit your manuscripts at

http://www.hindawi.com
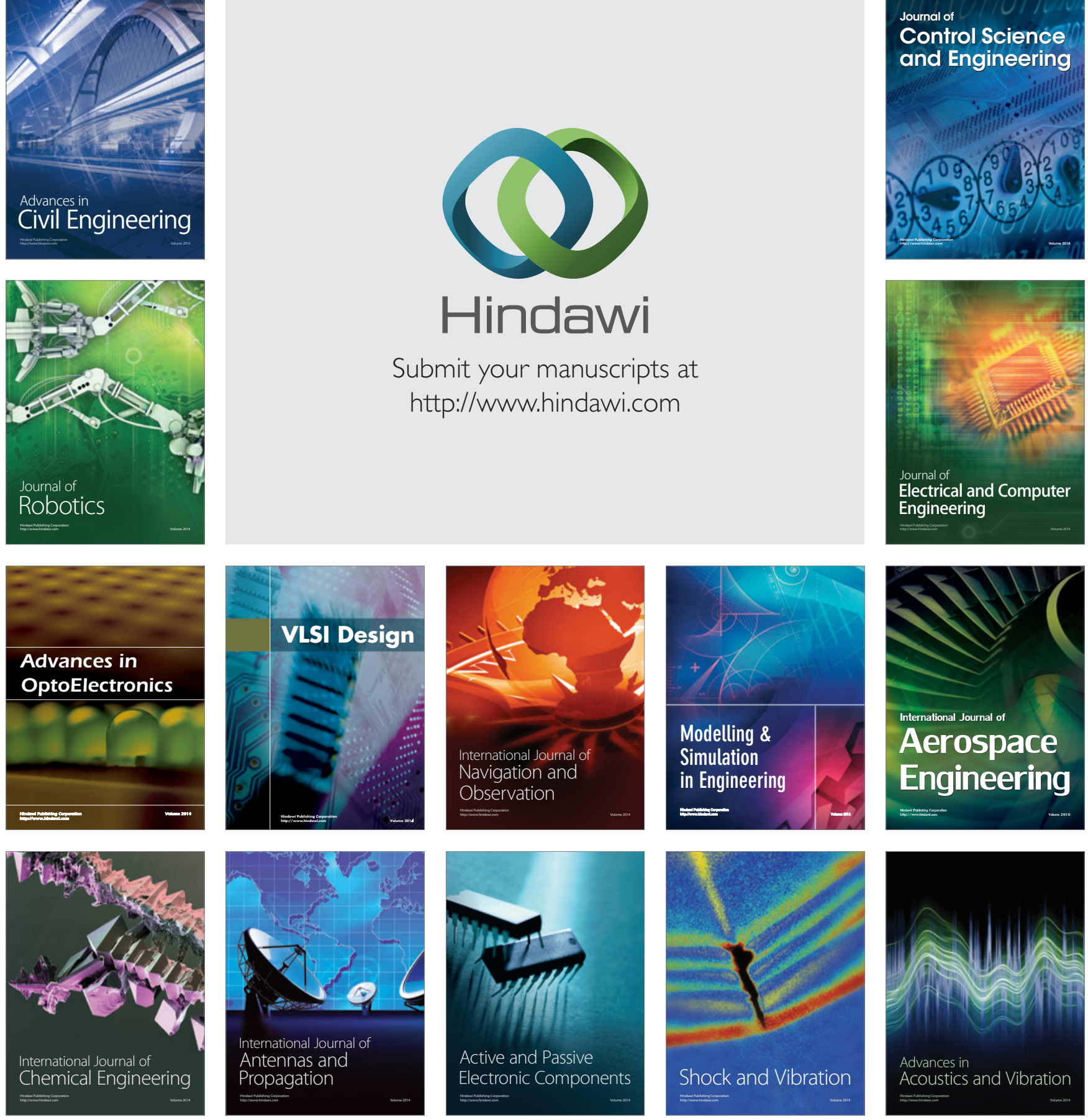